\title{
On the fractional $p$-Laplacian problems
}

\author{
Q-Heung Choi ${ }^{1}$ and Tacksun Jung ${ }^{2^{*}}$ (D)
}

${ }^{*}$ Correspondence:

tsjung@kunsan.ac.kr

${ }^{2}$ Department of Mathematics,

Kunsan National University, Kunsan

573-701, Korea

Full list of author information is

available at the end of the article

\begin{abstract}
This paper deals with nonlocal fractional $p$-Laplacian problems with difference. We get a theorem which shows existence of a sequence of weak solutions for a family of nonlocal fractional $p$-Laplacian problems with difference. We first show that there exists a sequence of weak solutions for these problems on the finite-dimensional subspace. We next show that there exists a limit sequence of a sequence of weak solutions for finite-dimensional problems, and this limit sequence is a sequence of the solutions of our problems. We get this result by the estimate of the energy functional and the compactness property of continuous embedding inclusions between some special spaces.
\end{abstract}

MSC: 35K05; 35K25; 35K35; 35K55; 35K92

Keywords: Nonlocal fractional $p$-Laplacian problems with difference; Fractional Laplace space; Fractional Sobolev space; Approximation method; Approximation weak solution; Limit sequence of the approximation weak solutions

\section{Introduction}

The nonlocal fractional $p$-Laplacian problems with difference appear in the models of nonlinear fractional Laplace flows such as the parabolic boundary value problems with time derivative and the fractional $p$-Laplacian differential operators. The fractional Laplacian flows arise in applications of nonlinear elasticity theory, electro rheological fluids, non-Newtonian fluid theory in a porous medium (cf. [9, 31, 40]).

In this paper we consider a family of the fractional $p$-Laplacian problems of Rothe type with difference under boundary and initial conditions:

$$
\begin{aligned}
& (-\Delta)_{g_{p}}^{s} u_{n}+\lambda V(x)\left|u_{n}\right|^{p-2} u_{n}+\frac{\left|u_{n}\right|^{r-1} u_{n}-\left|u_{n-1}\right|^{r-1} u_{n-1}}{h}=0 \text { in } \Omega, \\
& u_{n}=0 \quad \text { on } \partial \Omega, \\
& u_{n}(0)=u_{0} \quad \text { in } \Omega,
\end{aligned}
$$

where $\Omega$ is a bounded domain of $R^{N}, N \geq 3$, with smooth boundary $\partial \Omega, s \in(0,1), p$ is a real constant, $2 \leq p \leq N, r=p_{s}^{*}-1=\frac{N p}{N-s p}-1, g_{p}$ is a continuous function defined by $g_{p}(t)=|t|^{p-2} t, t \neq 0, g_{p}(0)=0, \lambda>0, V: \Omega \rightarrow[0, \infty)$ is a continuous function, and $u_{n}$ is a measurable function defined on $\Omega$ with valued into $R, n=1,2, \ldots$, and $(-\Delta)_{g_{p}}^{s}$ is the

(c) The Author(s) 2021. This article is licensed under a Creative Commons Attribution 4.0 International License, which permits use, sharing, adaptation, distribution and reproduction in any medium or format, as long as you give appropriate credit to the original author(s) and the source, provide a link to the Creative Commons licence, and indicate if changes were made. The images or other third party material in this article are included in the article's Creative Commons licence, unless indicated otherwise in a credit line to the material. If material is not included in the article's Creative Commons licence and your intended use is not permitted by statutory regulation or exceeds the permitted use, you will need to obtain permission directly from the copyright holder. To view a copy of this licence, visit http://creativecommons.org/licenses/by/4.0/. 
fractional $p$-Laplacian operator defined as follows: for each $x \in R^{N}$ and any $u \in C_{0}^{\infty}(\Omega)$,

$$
\begin{aligned}
(-\Delta)_{g_{p}}^{s} u(x) & =\text { P.V. } \int_{\Omega} g_{p}\left(\frac{|u(x)-u(y)|}{|x-y|^{s}}\right) \frac{u(x)-u(y)}{|u(x)-u(y)|} \frac{d y}{|x-y|^{N+s}} \\
& =\text { P.V. } \int_{\Omega} \frac{|u(x)-u(y)|^{p-2}}{|x-y|^{s(p-2)}} \frac{u(x)-u(y)}{|x-y|^{s}} \frac{u(x)-u(y)}{|u(x)-u(y)|} \frac{d y}{|x-y|^{N+s}},
\end{aligned}
$$

where P.V. denotes the Cauchy principle value. For $0<s<1,(-\Delta)_{g_{p}}^{s}$ is called the fractional $p$-Laplacian operator.

In the last years, for pure mathematical research and concrete real-world applications, the fractional $p$-Laplacian operator has been studied on the fractional Sobolev space

$$
W^{s} L_{g_{p}}(\Omega)=\left\{u \in L_{g_{p}}(\Omega): \int_{\Omega} \int_{\Omega} \frac{|u(x)-u(y)|^{p}}{|x-y|^{N+s p}} d x d y<\infty\right\}
$$

where $L_{g_{p}}(\Omega)$ is the Banach space defined by

$$
L_{g_{p}}(\Omega)=\left\{u: \Omega \rightarrow R \text { is a measurable function : } \int_{\Omega}\left(\int_{0}^{|u(x)|} g_{p}(t) d t\right) d x<\infty\right\} .
$$

The fractional $p$-Laplacian operator and the fractional Sobolev space arise in many fields of science, for example, elastic mechanics (see [40]), electro-rheological fluid dynamics(see [31]), and image processing (see [6]) and the references therein. When $0<s<1,(-\Delta)^{s}$ is the usual fractional Laplacian operator defined by: for each $x \in R^{N}$ and any $u \in C_{0}^{\infty}(\Omega)$,

$$
(-\Delta)^{s} u(x)=\operatorname{P.V} . \int_{\Omega} \frac{u(x)-u(y)}{|x-y|^{N+2 s}} \frac{u(x)-u(y)}{|u(x)-u(y)|} d y,
$$

where P.V. denotes the Cauchy principle value. Since $0<s<1,(-\Delta)^{s}$ is called the fractional Laplacian operator. For the fractional Laplacian operator, see $[8,10,19]$ and the references therein. The fractional Laplacian problems arise from continuum mechanics, phase transition phenomena, population dynamics, minimum surfaces, and game theory. The body of literature on the fractional Laplacian operators and their applications is quite large. We refer the reader to $[3,12,13,24-29,33-38]$ and the references therein. For the basic properties of the fractional Sobolev spaces, we refer the readers to [10]. If $s \rightarrow 1^{-}$, $(-\Delta)^{\frac{s(-)}{2}}$ reduces to $-\Delta$. For $s=1$, we identify $(-\Delta)^{s}$ with the classical Laplacian operator $-\Delta$. If $2<s<\infty$, (1.1) is called $s$-exponent problems of elliptic type. The $s$-exponent Laplacian problems of elliptic type appear in a lot of applications, for example, elastic mechanics, electro-rheological fluid dynamics, and image processing. We refer the readers to $[2,9,11,22,23,31]$ and the references therein. In $[5,6,16]$, there are some papers concerning related equations involving the fractional Laplacian operator, but results for fractional Sobolev spaces and the fractional Laplacian operator with exponent are few. In particular, the fractional Laplacian operator with variable exponent was suggested firstly by Lorenzo and Hartley [20]. The fractional Laplacian operator with variable exponent and the variable exponent fractional Sobolev space have appeared in a nonlinear diffusion process. Some diffusion processes reacting to temperature changes can be explained well by fractional derivatives in a nonlocal integro differential operator (see [21]). In $[17,18,30]$, the authors consider the pseudodifferential equations on the fractional Sobolev spaces. 
In recent years, the Kirchhoff equations involving fractional $p$-Laplacian have attracted interest and have been researched by some mathematicians. In particular, when $s=1$ and $p=2,-\Delta$ is the classical Laplace operator. Ji, Fang, and Zhang [15] provided multiplicity results of solutions for asymptotically linear Kirchhoff equations by using a variant version of mountain pass theorem and the variational method. When $0<s<1$ and $p=2$, Fiscella [14] provided the existence of solution for a class of Kirchhoff-type problems involving fractional Laplacian operator singular term and a critical nonlinearity. When $0<s<1$ and $1<p<N / s$, Xiang, Zhang, and Rădulescu [39] obtained multiplicity results for superlinear Schrödinger-Kirchhoff equations involving fractional $\mathrm{N} / \mathrm{s}$-Laplacian with critical exponential nonlinearity by using the concentration compactness principle in the fractional Sobolev and mountain pass theorem. When $0<s<1$ and $p=N / s$, Mingqi, Rădulescu, and Zhang [25] provided existence and multiplicity of solutions for Kirchhoff equations involving fractional $N / s$-Laplacian with critical nonlinearity by the mountain pass geometry and Ekeland's variational principle. They [26] also obtained the existence and multiplicity results of solutions for Kirchhoff equations involving fractional $\mathrm{N} / \mathrm{s}$-Laplacian with singular exponential nonlinearity by using the same methods.

The weak solutions $u_{n} \in W^{s} L_{g_{p}}(\Omega)$ of (1.1) are a measurable function defined on $\Omega$ with valued into $R, n=1,2, \ldots$, and satisfy the following in weak sense:

$$
\int_{\Omega}\left[(-\Delta)_{g_{p}}^{s} u_{n}+\lambda V(x)\left|u_{n}\right|^{p-2} u_{n}\right] w(x) d x+\int_{\Omega} \frac{\left|u_{n}\right|^{r-1} u_{n}-\left|u_{n-1}\right|^{r-1} u_{n-1}}{h} w(x) d x=0
$$

for any $w \in W^{s} L_{g_{p}}(\Omega)$ and

$$
\left\|u(x)-u_{0}\right\| \rightarrow 0 \quad \text { as } x \rightarrow \theta
$$

Our main result is as follows.

Theorem 1.1 Assume that $0<s<1,2 \leq p<\infty, r=\frac{N p}{N-s p}-1=p_{s}^{*}-1, N>s p u_{0} \in W^{s} L_{g_{p}}(\Omega)$ and $V: \bar{\Omega} \rightarrow[0, \infty)$ is a continuous function satisfying

$\left(V_{1}\right) J=\operatorname{int}\left(V^{-1}(0)\right) \subset \Omega$ is a nonempty bounded domain and $\bar{J}=V^{-1}(0)$,

$\left(V_{2}\right)$ there exists a nonempty open domain $\Omega_{0} \subset J$ such that $V(x)=0$ for all $x \in \bar{\Omega}_{0}$.

Then there exists a family of weak solutions $u_{n} \in W^{s} L_{g_{p}}(\Omega), n=1,2, \ldots$ of $(1.1)$.

The outline of the proof of Theorem 1.1 is as follows: We first prove the existence of a sequence of weak solutions for a family of the fractional $p$-Laplacian difference equations defined on the finite-dimensional subspace. We next show that there exists a limit sequence of the sequence of weak solutions for the finite-dimensional problem, and this limit sequence is the sequence of the solutions of our problem. We get this result by the estimate of the energy functional and the compactness property of the continuous embedding inclusions between some special spaces. In Sect. 2, we introduce the fractional Lebesgue space with exponent and the fractional Sobolev space and give some properties. In Sect. 3, we first prove that problem (1.1) defined on the finite-dimensional subspace has a sequence of weak solutions for each $n=1,2, \ldots$ In Sect. 4 , we show that there exists a limit sequence of the sequence of weak solutions for finite-dimensional problem, and this limit sequence is a sequence of solutions of our problem (1.1). 


\section{Preliminaries}

For the variational setting for our problem, we introduce some definitions and theories on the fractional Lebesgue space with exponent and the fractional Sobolev space.

Let $N \geq 3$ and $\Omega$ be a bounded open domain in $R^{N}$ with smooth boundary $\partial \Omega$. Let $2 \leq p<\infty$ and $r=\frac{N p}{N-s p}-1$. The Lebesgue space with $p$-exponent is

$$
L^{p}(\Omega)=\left\{u: \Omega \rightarrow R \text { is a measurable function }:\left(\int_{\Omega}|u(x)|^{p} d x\right)^{\frac{1}{p}}<\infty\right\}
$$

with the Luxemburg norm on $L^{p}(\Omega)$

$$
\|u\|_{L^{p}(\Omega)}=\inf \left\{\lambda>\left.0\left|\int_{\Omega}\right| \frac{u(x)}{\lambda}\right|^{p} d x \leq 1\right\}
$$

The Sobolev space with $p$-exponent is

$$
\begin{gathered}
W^{1, p}(\Omega)=\left\{u \in L^{p}(\Omega): \Omega \rightarrow R\right. \text { is a measurable function : } \\
\left.[u]_{W^{1, p}(\Omega)}=\left(\int_{\Omega}|\nabla u(x)|^{p} d x\right)^{\frac{1}{p}}<\infty\right\}
\end{gathered}
$$

with the Sobolev norm

$$
\|u\|_{W^{1, p}(\Omega)}=\left[\|u\|_{L^{p}(\Omega)}^{p}+[u]_{W^{1, p}(\Omega)}^{p}\right]^{\frac{1}{p}} .
$$

Then $L^{p}(\Omega)$ and $W^{1, p}(\Omega)$ are Banach spaces. We also define the Sobolev space $W_{0}^{1, p}(\Omega)$ as the closure of $C_{0}^{\infty}(\Omega)$ in $W^{1, p}(\Omega)$. The space is also a reflexive Banach space. If $p$ is bounded, then norm $\|\cdot\|_{W^{1, p}(\Omega)}$ is equivalent to the norm $[\cdot]_{W^{1, p}(\Omega)}$. If $p=\infty, L^{\infty}(\Omega)$ is the Banach space of essentially bounded. If $p$ is bounded and $p^{\prime}$ is the conjugate exponent of $p$ defined by $p^{\prime}=\frac{p}{p-1}$, then the dual space $\left(L^{p}(\Omega)\right)^{\prime}$ can be identified with $L^{p^{\prime}}(\Omega)$. If $1<p<$ $\infty$, then the Lebesgue space $L^{p}(\Omega)$ with $p$-exponent is separable and reflexive. In $L^{p}(\Omega)$, Hölder's inequality is valid: for all $u \in L^{p}(\Omega), v \in L^{p^{\prime}}(\Omega)$ with $1<p<\infty$, we have

$$
\int_{\Omega} u v d x \leq 2\|u\|_{L^{p}(\Omega)}\|v\|_{L^{p^{\prime}}(\Omega)}
$$

Let us set $g_{p}(t)=|t|^{p-2} t$. Then $g_{p}^{-1}(t)=t^{\frac{1}{p-1}}$ if $t>0, g_{p}^{-1}(t)=-t^{\frac{1}{p-1}}$ if $t<0$ and $g_{p}^{-1}(0)=0$. By Young's inequality, we have

$$
r t \leq \frac{1}{p}|r|^{p}+\frac{1}{p^{\prime}}|t|^{p^{\prime}}=\int_{0}^{r}|x|^{p-2} x d x+\int_{0}^{t} y^{\frac{1}{p-1}} d y \quad \text { for all } r, t \geq 0 .
$$

Let $L_{g_{p}}(\Omega)$ be the space defined by

$$
\begin{aligned}
L_{g_{p}}(\Omega)= & \{u: \Omega \rightarrow R \text { is a measurable such that } \\
& \left.\sup \left\{\int_{\Omega} u v d x ; \int_{\Omega}\left[\int_{0}^{|\nu|} y^{\frac{1}{p-1}} d y\right] d x \leq 1\right\}<\infty\right\}
\end{aligned}
$$


equipped with the norm

$$
\|u\|_{L_{g_{p}}}=\sup \left\{\int_{\Omega} u v d x ; \int_{\Omega}\left[\int_{0}^{|v|} y^{\frac{1}{p-1}} d y\right] d x \leq 1\right\} .
$$

Then $\left(L_{g_{p}}(\Omega),\|u\|_{L_{g_{p}}}\right)$ is a Banach space whose norm is equivalent to the Luxemburg norm

$$
\|u\|_{g_{p}}=\inf \left\{\lambda>0 ; \int_{\Omega} \int_{0}^{\left|\frac{u(x)}{\lambda}\right|}|t|^{p-2} t d t d x \leq 1\right\} .
$$

In $L_{g_{p}}(\Omega)$, Hölder's inequality is valid: for all $u \in L_{g_{p}}(\Omega), v \in L_{g_{p}^{-1}}(\Omega)$ with $1<p<\infty$, we have

$$
\int_{\Omega}|u v| d x \leq 2\|u\|_{g_{p}}\|v\|_{g_{p}^{-1}}
$$

Now we introduce the fractional Sobolev space with $p$-exponent. Let $0<s<1$ and $2 \leq p<$ $\infty$. The fractional Sobolev space with $p$-exponent is defined by

$$
W^{s} L_{g_{p}}(\Omega)=\left\{u \in L_{g_{p}}(\Omega):[u]_{s, g_{p}}^{p}=\int_{\Omega} \int_{\Omega}\left[\int_{0}^{\frac{|u(x)-u(y)|}{|x-y|^{s}}}|t|^{p-2} t d t\right] \frac{d x d y}{|x-y|^{N}}<\infty\right\}
$$

equipped with the norm

$$
\|u\|_{s, g_{p}}=\left(\|u\|_{g_{p}}^{p}(\Omega)+[u]_{s, g_{p}}^{p}\right)^{\frac{1}{p}} .
$$

Lemma 2.1 ([7]) Let $0<s<1$ and $2 \leq p<\infty$. Then $W^{s} L_{g_{p}}\left(R^{N}\right)$ is a reflexive and separable Banach space. Moreover, $C_{0}^{\infty}\left(R^{N}\right)$ is dense in $W^{s} L_{g_{p}}\left(R^{N}\right)$.

Let $W_{0}^{s} L_{g_{p}}(\Omega)$ denote the closure of $C_{0}^{\infty}(\Omega)$ in the norm $\|u\|_{s, g_{p}}$.

The following lemma shows that the norm $[\cdot]_{s, g_{p}}$ is a norm of $W^{s} L_{g_{p}}(\Omega)$ equivalent to $\|\cdot\|_{s, g_{p}}$.

Lemma 2.2 ([32]; Generalized Poincaré inequality) Let $0<s<1$ and $2 \leq p<\infty$. Then there exists a positive constant $C>0$ such that

$$
\|u\|_{g_{p}} \leq[u]_{s, g_{p}}, \quad \forall u \in W_{0}^{S} L_{g_{p}}(\Omega)
$$

That is, the embedding

$$
W_{0}^{s} L_{g_{p}}(\Omega) \hookrightarrow L_{g_{p}}(\Omega)
$$

is continuous and compact. Furthermore, $[u]_{s, g_{p}}$ is a norm of $W_{0}^{s} L_{g_{p}}(\Omega)$ equivalent to $\|\cdot\|_{s, g_{p}}$.

Lemma 2.3 Let $0<s<1,2 \leq p<\infty$. If $N>s p$, for any fixed constant exponent $q \in\left(1, \frac{N p}{N-s p}\right]$, $W_{0}^{s} L_{g_{p}}(\Omega)$ is continuously embedded into $L_{g_{q}}(\Omega)$. If $u \in W_{0}^{s} L_{g_{p}}(\Omega)$, then there exists a constant $C_{1}=C_{1}(N, p, q, s)>0$ such that

$$
\|u\|_{g_{q}} \leq C_{1}\|u\|_{s, p} .
$$


Furthermore, $[u]_{s, p}$ is a norm of $W_{0}^{s} L_{g_{p}}(\Omega)$. Moreover, there exists a constant $C_{2}=$ $C_{2}(N, p, s)>0$ such that

$$
\|u\|_{g_{q}} \leq C_{2}[u]_{s, p}
$$

Proof By Theorem 6.7 and Theorem 6.9 of [10], for $N>s p$ and any fixed constant exponent $q \in\left(1, \frac{N p}{N-s p}\right], W_{0}^{s} L_{g_{p}}(\Omega)$ is continuously embedded into $L_{g_{q}}(\Omega)$. It follows that (2.2) holds. By combining inequalities (2.1) and (2.2), $[u]_{s, g_{p}}$ is an equivalent norm of $W_{0}^{s} L_{g_{p}}(\Omega)$. It follows that (2.3) holds.

Lemma 2.4 Let $0<s_{1}<s<s_{2}<1$ and $2 \leq p<\infty$. Then the embeddings

$$
W_{0}^{s_{2}} L_{g_{p}}(\Omega) \hookrightarrow W_{0}^{s} L_{g_{p}}(\Omega) \hookrightarrow W_{0}^{s_{1}} L_{g_{p}}(\Omega)
$$

are continuous.

Proof For any $u \in W_{0}^{s_{2}} L_{g_{p}}(\Omega)$, we have

$$
\begin{aligned}
& \int_{\Omega} \int_{\Omega \cap\{|x-y| \geq 1\}}\left[\int_{0}^{\frac{|u(x)|}{|x-y|^{s}}}|t|^{p-2} t d t\right] \frac{d x d y}{|x-y|^{N}} \\
& \quad \leq \int_{\Omega} \int_{\Omega \cap\{|x-y| \geq 1\}}\left[\int_{0}^{\frac{|u(x)|}{|x-y|^{S 1}}}|t|^{p-2} t d t\right] \frac{d x d y}{|x-y|^{N}} \\
& \leq \int_{\Omega} \int_{\Omega \cap\{|z| \geq 1\}}\left[\int_{0}^{\frac{|u(x)|}{|z|^{S 1}}}|t|^{p-2} t d t\right] \frac{d x d z}{|x-y|^{N}} \\
& \quad \leq C \int_{\Omega}|u(x)|^{p} d x .
\end{aligned}
$$

Moreover we have

$$
\begin{aligned}
& \int_{\Omega} \int_{\Omega \cap\{|x-y| \leq 1\}}\left[\int_{0}^{\frac{|u(x)-u(y)|}{|x-y|^{S}}}|t|^{p-2} t d t\right] \frac{d x d y}{|x-y|^{N}} \\
& \quad \leq \int_{\Omega} \int_{\Omega \cap\{|x-y| \leq 1\}}\left[\int_{0}^{\frac{|u(x)-u(y)|}{|x-y|^{S 2}}}|t|^{p-2} t d t\right] \frac{d x d y}{|x-y|^{N}} \\
& \quad \leq \int_{\Omega} \int_{\Omega}\left[\int_{0}^{\frac{|u(x)-u(y)|}{|x-y|^{S 2}}}|t|^{p-2} t d t\right] \frac{d x d y}{|x-y|^{N}} .
\end{aligned}
$$

Thus we have

$$
\begin{aligned}
\int_{\Omega} \int_{\Omega} & {\left[\int_{0}^{\frac{|u(x)-u(y)|}{|x-y|^{S}}}|t|^{p-2} t d t\right] \frac{d x d y}{|x-y|^{N}} } \\
\leq & \int_{\Omega} \int_{\Omega \cap\{|x-y| \geq 1\}}\left[\int_{0}^{\frac{|u(x)-u(y)|}{|x-y|^{S}}}|t|^{p-2} t d t\right] \frac{d x d y}{|x-y|^{N}} \\
& +\int_{\Omega} \int_{\Omega \cap\{|x-y| \leq 1\}}\left[\int_{0}^{\frac{|u(x)-u(y)|}{|x-y|^{S}}}|t|^{p-2} t d t\right] \frac{d x d y}{|x-y|^{N}}
\end{aligned}
$$




$$
\begin{aligned}
& \leq C \int_{\Omega}|u(x)|^{p} d x+\int_{\Omega} \int_{\Omega}\left[\int_{0}^{\frac{|u(x)-u(y)|}{|x-y|^{S}}}|t|^{p-2} t d t\right] \frac{d x d y}{|x-y|^{N}} \\
& \leq C\left(\int_{\Omega}|u(x)|^{p} d x+\int_{\Omega} \int_{\Omega}\left[\int_{0}^{\frac{|u(x)-u(y)|}{|x-y|^{S^{2}}}}|t|^{p-2} t d t\right] \frac{d x d y}{|x-y|^{N}}\right) .
\end{aligned}
$$

It follows from this inequality that we can easily verify that the embedding $W_{0}^{s_{2}} L_{g_{p}}(\Omega) \hookrightarrow$ $W_{0}^{s} L_{g_{p}}(\Omega)$ is continuous. Similarly, for any $u \in W_{0}^{s} L_{g_{p}}(\Omega)$, we have

$$
\begin{aligned}
& \int_{\Omega} \int_{\Omega \cap\{|x-y| \geq 1\}}\left[\int_{0}^{\frac{|u(x)|}{|x-y|^{S}}}|t|^{p-2} t d t\right] \frac{d x d y}{|x-y|^{N}} \\
& \leq \int_{\Omega} \int_{\Omega \cap\{|z| \geq 1\}}\left[\int_{0}^{\frac{|u(x)|}{|z|^{S 1}}}|t|^{p-2} t d t\right] \frac{d x d y}{|x-y|^{N}} \\
& \leq D \int_{\Omega}|u(x)|^{p} d x .
\end{aligned}
$$

Moreover, we have

$$
\begin{aligned}
& \int_{\Omega} \int_{\Omega \cap\{|x-y| \leq 1\}}\left[\int_{0}^{\frac{|u(x)-u(y)|}{\left.|x-y|\right|^{1}}}|t|^{p-2} t d t\right] \frac{d x d y}{|x-y|^{N}} \\
& \quad \leq \int_{\Omega} \int_{\Omega \cap\{|x-y| \leq 1\}}\left[\int_{0}^{\frac{|u(x)-u(y)|}{|x-y|^{s}}}|t|^{p-2} t d t\right] \frac{d x d y}{|x-y|^{N}} \\
& \quad \leq \int_{\Omega} \int_{\Omega}\left[\int_{0}^{\frac{|u(x)-u(y)|}{|x-y|^{S}}}|t|^{p-2} t d t\right] \frac{d x d y}{|x-y|^{N}} .
\end{aligned}
$$

Thus we have

$$
\begin{aligned}
& \int_{\Omega} \int_{\Omega}\left[\int_{0}^{\frac{|u(x)-u(y)|}{|x-y|^{S} \mid}}|t|^{p-2} t d t\right] \frac{d x d y}{|x-y|^{N}} \\
& \leq \int_{\Omega} \int_{\Omega \cap\{|x-y| \geq 1\}}\left[\int_{0}^{\frac{|u(x)-u(y)|}{|x-y|^{S 1}}}|t|^{p-2} t d t\right] \frac{d x d y}{|x-y|^{N}} \\
& \quad+\int_{\Omega} \int_{\Omega \cap\{|x-y| \leq 1\}}\left[\int_{0}^{\frac{|u(x)-u(y)|}{|x-y|^{S} \mid}}|t|^{p-2} t d t\right] \frac{d x d y}{|x-y|^{N}} \\
& \leq D \int_{\Omega}|u(x)|^{p} d x+\int_{\Omega} \int_{\Omega}\left[\int_{0}^{\frac{|u(x)-u(y)|}{|x-y|^{S}}}|t|^{p-2} t d t\right] \frac{d x d y}{|x-y|^{N}} \\
& \leq D\left(\int_{\Omega}|u(x)|^{p} d x+\int_{\Omega} \int_{\Omega}\left[\int_{0}^{\frac{|u(x)-u(y)|}{|x-y|^{s}}}|t|^{p-2} t d t\right] \frac{d x d y}{|x-y|^{N}}\right) .
\end{aligned}
$$

It follows that the embedding $W_{0}^{s} L_{g_{p}}(\Omega) \hookrightarrow W_{0}^{s_{1}} L_{g_{p}}(\Omega)$ is continuous. Thus the proof of the lemma is complete.

Lemma 2.5 Let $0<s<1,2 \leq p<\infty$. If $N>s p$, for any fixed constant exponent $q \in\left(1, \frac{N p}{N-s p}\right]$, the embedding

$$
W^{s} L_{g_{p}}(\Omega) \hookrightarrow L_{g_{q}}(\Omega)
$$


is continuous and compact. That is, if $u \in W^{s} L_{g_{p}}(\Omega)$, then there exists a constant $D=$ $D(N, p, s)>0$ such that

$$
\|u\|_{g_{q}} \leq D\|u\|_{s, g_{p}} .
$$

Furthermore, $[u]_{s, g_{p}}$ is a norm of $W_{0}^{s} L_{g_{p}}(\Omega)$. Moreover, there exists a constant $C_{2}=$ $C_{2}(N, p, s)>0$ such that

$$
\|u\|_{g_{q}} \leq C_{2}[u]_{s, g_{p}} .
$$

Proof Let us set

$$
\begin{aligned}
& p_{s}^{*}=\frac{N p}{N-s p}, \\
& \|u\|_{g_{p_{s}^{*}}}\left(V_{i}\right)=\left.\|u\|_{g_{p_{s}^{*}}}\right|_{V_{i}}, \quad[u]_{s, g_{p}}\left(V_{i}\right)=\left.[u]_{s, g_{p}}\right|_{V_{i}}, \quad \text { and } \quad\|u\|_{s, g_{p}}\left(V_{i}\right)=\left.\|u\|_{s, g_{p}}\right|_{V_{i}} .
\end{aligned}
$$

Since $0<s<1$ and $N>s p$, there exists a constant $\tau_{1}>0$ such that

$$
p_{s}^{*}-p \geq \tau_{1}>0 .
$$

Since $\Omega$ is bounded, there exist a constant $\epsilon>0$ and $l$ numbers of disjoint hypercubes $V_{i}$ such that $\Omega=\bigcup_{i=1}^{l} V_{i}$ and diam $V_{i}<\epsilon$ such that

$$
p_{s}^{*}-p \geq \frac{\tau_{1}}{2}>0
$$

on $V_{i}, i=1,2, \ldots, l$. By Lemma 2.3, Theorem 6.7 , and Theorem 6.9 of [12], there exists a constant $D=D(N, s, p)$ such that

$$
\|u\|_{g_{p_{s}^{*}}^{*}}\left(V_{i}\right) \leq D\left(\|u\|_{g_{p}}^{p}\left(V_{i}\right)+[u]_{s, g_{p}}^{p}\left(V_{i}\right)\right)^{\frac{1}{p}} .
$$

By Hölder's inequality, if $q \in\left(1, p_{s}^{*}\right]$, we have

$$
\|u\|_{g_{q}}\left(V_{i}\right) \leq D\|u\|_{g_{p_{s}^{*}}}\left(V_{i}\right) .
$$

We note that

$$
\|u\|_{g_{q}}(\Omega) \leq \sum_{i=1}^{l}\|u\|_{g_{q}}\left(V_{i}\right) .
$$

Thus we have

$$
\|u\|_{g_{q}}(\Omega) \leq \sum_{i=1}^{l}\|u\|_{g_{q}}\left(V_{i}\right) \leq D \sum_{i=1}^{l}\|u\|_{g_{p_{s}^{*}}^{*}}\left(V_{i}\right) .
$$

It follows from (2.4) that

$$
\|u\|_{g_{q}}(\Omega) \leq \sum_{i=1}^{l} D\left(\|u\|_{g_{p}}^{p}\left(V_{i}\right)+[u]_{s, p}^{p}\left(V_{i}\right)\right)^{\frac{1}{p}} \leq D\|u\|_{s, p} .
$$


Thus the embedding $W^{s} L_{g_{p}}(\Omega) \hookrightarrow L_{g_{q}}(\Omega)$ is continuous. Furthermore, we show that the embedding is compact. In fact, in the constant $p_{s}^{*}$ on $V_{i}$, for $q \in\left(1, p_{s}^{*}\right]$ on $V_{i}$, the embedding $W^{s} L_{g_{p}}\left(V_{i}\right) \hookrightarrow L_{g_{q}}\left(V_{i}\right)$ is compact. Thus the embedding $W^{s} L_{g_{p}}(\Omega) \hookrightarrow L_{g_{q}}(\Omega)$ is compact. It follows that there exists a constant $D=D(N, p, s)>0$ such that

$$
\|u\|_{g_{q}} \leq D\|u\|_{s, g_{p}} .
$$

By Lemma 2.2, we have the following lemma.

Lemma 2.6 If $u, u_{n} \in W_{0}^{s} L_{g_{p}}(\Omega), n=1,2, \ldots$, then the following statements are equivalent to each other:

(i) $\lim _{n \rightarrow \infty}\left\|u_{n}-u\right\|_{s, g_{p}}=0, i=1,2$,

(ii) $\lim _{n \rightarrow \infty} \int_{\Omega}\left|u_{n}-u\right|^{p} d x=0$ and $\lim _{n \rightarrow \infty}\left[u_{n}-u\right]_{s, g_{p}}=0$,

(iii) $u_{n} \rightarrow u$ in measure in $\Omega$ and $\lim _{n \rightarrow \infty} \int_{\Omega}\left|u_{n}\right|^{p} d x=\int_{\Omega}|u|^{p} d x$.

We need the following inequality for the $p$-Laplacian operator.

Lemma 2.7 ([1]) Let $1<p<\infty$. Then there exist constants $C_{1}$ and $C_{2}$ depending on $p$ and $N$ such that, for any $\xi, \eta \in R^{N}$,

(i) $\left.|| \xi\right|^{p-2} \xi-|\eta|^{p-2} \eta\left|\leq C_{1}(|\xi|+|\eta|)^{p-2}\right| \xi-\eta \mid$

and

(ii) $\quad\left(|\xi|^{p-2} \xi-|\eta|^{p-2} \eta\right) \cdot(\xi-\eta) \geq C_{2}|\xi-\eta|^{p}$.

We recall a fundamental fact, which is a crucial role for our main result.

Lemma 2.8 ([4]) Assume that $Q$ is a continuous vector field from $R^{N}$ to $R^{N}$ and satisfies

$$
Q(x) \cdot x \geq 0 \quad \text { if }|x|=\rho
$$

for some $\rho>0$. Then there exists a point $x \in B_{\rho}(0)$ such that

$$
Q(x)=0
$$

where $B_{\rho}(0)$ denotes a ball centered at the origin with radius $\rho$ in $R^{N}$.

\section{Existence of approximating solutions}

In this section we show that there exists a unique approximating solution for (1.1) on each finite-dimensional subspace.

Let us choose a family of bases $\left\{\phi_{i}(x)\right\}_{i=1}^{\infty}$ in $W_{0}^{s} L_{g_{p}}(\Omega)$ such that $\left\{\phi_{i}(x)\right\}_{i=1}^{\infty}$ is an orthonormal system in $L^{p}(\Omega)$ and $\operatorname{span}\left\{\phi_{i}(x)\right\}_{i=1}^{\infty}$ is dense in $W_{0}^{s} L_{g_{p}}(\Omega)$. Let $u_{0} \in W_{0}^{s} L_{g_{p}}(\Omega)$. Since $\operatorname{span}\left\{\phi_{i}(x)\right\}_{i=1}^{\infty}$ is dense in $W_{0}^{s} L_{g_{p}}(\Omega)$, any element $u_{n}$ in $W_{0}^{s} L_{g_{p}}(\Omega)$ and the initial data $u_{0}$ can be expanded as

$$
u_{n}(x)=\sum_{i=1}^{\infty} a_{n}^{i} \phi_{i}(x), \quad u_{0}(x)=\sum_{i=1}^{\infty} a_{0}^{i} \phi_{i}(x) .
$$


Let us define the finite subspace $F_{k}$ of $W_{0}^{s} L_{g_{p}}(\Omega)$ by

$$
F_{k}=\operatorname{span}\left\{\phi_{1}(x), \ldots, \phi_{k}(x)\right\}
$$

Let $N$ be any positive integer which shall be sent to infinity and $h$ be any small positive number. For any fixed integer $k=1,2, \ldots$, let $u_{n, k}=\sum_{i=1}^{k} a_{n, k}^{i} \phi_{i}(x)$ be a family of the Galerkin approximating solutions for a family of fractional Laplace equations with $p$-exponent and difference defined on the finite-dimensional subspaces.

$$
\begin{gathered}
\int_{\Omega}(-\Delta)_{g_{p}}^{s} u_{n, k} \cdot \psi d x+\lambda \int_{\Omega} V(x)\left|u_{n, k}\right|^{p-2} u_{n, k} \cdot \psi d x \\
+\int_{\Omega} \frac{\left|u_{n, k}\right|^{r-1} u_{n, k}-\left|u_{n-1}\right|^{r-1} u_{n-1}}{h} \cdot \psi d x=0,
\end{gathered}
$$

$\forall \psi \in F_{k}$,

$$
u_{0, k}(x)=\sum_{i=1}^{k} a_{0}^{i} \phi_{i}(x) .
$$

Now we shall show that, for each $n=1,2, \ldots, N$ and $k=1,2, \ldots,(3.1)$ has a unique solution $u_{n, k} \in F_{k} \subset W_{0}^{s} L_{g_{p}}(\Omega)$.

Lemma 3.1 (Existence of approximating weak solutions) For each $n=1,2, \ldots, N$ and $k=$ $1,2, \ldots$, there exists a unique weak solution $u_{n, k} \in F_{k} \subset W_{0}^{s} L_{g_{p}}(\Omega)$ of (3.1).

Proof Let us choose any element $v \in F_{k} \subset W_{0}^{s} L_{g_{p}}(\Omega)$. Then $v$ can be represented by

$$
\nu=\sum_{i=1}^{k} \rho^{i} \phi_{i} .
$$

Let us set

$$
\rho=\left(\rho^{1}, \ldots, \rho^{k}\right) \in R^{k}
$$

Let us define the functional $J_{n, k}^{i}(\rho)$ by

$$
\begin{aligned}
J_{n, k}^{i}(\rho)= & \int_{\Omega}(-\Delta)_{g_{p}}^{s} \nu \cdot \phi_{i} d x+\lambda \int_{\Omega} V(x)|v|^{p-2} v \cdot \phi_{i} d x+\int_{\Omega} \frac{|v|^{r-1} v-\left|u_{n-1}\right|^{r-1} u_{n-1}}{h} \cdot \phi_{i} d x \\
= & \int_{\Omega} \int_{\Omega}\left|\frac{|v(x)-v(y)|}{|x-y|^{s}}\right|^{p-2} \frac{v(x)-v(y)}{|x-y|^{s}} \frac{\phi_{i}(x)-\phi_{i}(y)}{|x-y|^{N+s}} d x d y \\
& +\lambda \int_{\Omega} V(x)|v|^{p-2} v \cdot \phi_{i} d x+\int_{\Omega} \frac{|v|^{r-1} v-\left|u_{n-1}\right|^{r-1} u_{n-1}}{h} \cdot v d x .
\end{aligned}
$$

Let us define the functional $J_{n, k}=\left(J_{n, k}^{1}, \ldots, J_{n, k}^{k}\right): R^{k} \rightarrow R^{k}$. Then $J_{n, k}$ is continuous on $\rho$ and satisfies

$$
J_{n, k}(\rho) \cdot \rho=\sum_{i=1}^{k} J_{n, k}^{i} \rho^{i}
$$




$$
\begin{aligned}
= & \int_{\Omega} \int_{\Omega} \frac{|v(x)-v(y)|^{p}}{|x-y|^{N+s p}} d x d y \\
& +\lambda \int_{\Omega} V(x)|v|^{p} d x+\int_{\Omega} \frac{|v|^{r-1} v-\left|u_{n-1}\right|^{r-1} u_{n-1}}{h} \cdot v d x .
\end{aligned}
$$

We claim that $J_{n, k}(\rho) \cdot \rho \geq 0$. In fact, by Young's inequality and generalized Poincarés inequality of Lemma 2.2, for any $\epsilon>0$, there exists a constant $C_{\epsilon}>0$ such that

$$
\begin{aligned}
\int_{\Omega}\left|u_{n-1}\right|^{r-1} u_{n-1} \cdot v d x & \leq \frac{p-1}{p} \int_{\Omega}\left|u_{n-1}\right|^{\frac{r p}{p-1}} d x+\frac{1}{p} \int_{\Omega}|v|^{p} d x \\
& \leq C_{\epsilon} \int_{\Omega}\left|u_{n-1}\right|^{\frac{r p}{p-1}} d x+\epsilon[v]_{s, g_{p}}^{p} .
\end{aligned}
$$

Thus

$$
\begin{gathered}
J_{h, k}(\rho) \cdot \rho \geq[v]_{s, g_{p}}^{p}+\lambda \int_{\Omega} V(x)|v|^{p} d x+\frac{1}{h} \int_{\Omega}|v|^{r+1} d x \\
-\frac{C_{\epsilon}}{h} \int_{\Omega}\left|u_{n-1}\right|^{\frac{r p}{p-1}} d x-\frac{\epsilon}{h}[v]_{s, g_{p}}^{p} .
\end{gathered}
$$

By (3.2) and (3.3), we have

$$
J_{n, k}(\rho) \cdot \rho \geq\left(1-\frac{\epsilon}{h}\right)[v]_{s, g_{p}}^{p}+\lambda \int_{\Omega} V(x)|v|^{p} d x+\frac{1}{h} \int_{\Omega}|v|^{r+1} d x-\frac{C_{\epsilon}}{h} \int_{\Omega}\left|u_{n-1}\right|^{\frac{r p}{p-1}} d x .
$$

Taking $\epsilon>0$ so that $\epsilon=\frac{h}{2}$ for a small positive number $h$, we have

$$
J_{n, k}(\rho) \cdot \rho \geq \frac{1}{2}[v]_{s, g_{p}}^{p}+\lambda \int_{\Omega} V(x)|v|^{p} d x+\frac{1}{h} \int_{\Omega}|v|^{r+1} d x-\frac{C_{\epsilon}}{h} \int_{\Omega}\left|u_{n-1}\right|^{\frac{r p}{p-1}} d x
$$

By Hölder's inequality and the orthonormality of $\left\{\phi_{i}\right\}$ in $L_{g_{p}}(\Omega)$, we have

$$
\left(\int_{\Omega}|\nu|^{r+1} d x\right)^{\frac{2}{r+1}} \geq \frac{1}{|\Omega|^{\frac{r-1}{r+1}}}|\rho|^{2} .
$$

Since $r+1=p_{s}^{*}>p$ and $\lambda>0$, we can choose $|\rho|^{2}$ so large that the right-hand side of (3.4) is nonnegative. It follows from Lemma 2.8 that $J_{n, k}$ has a zero. Let this point be $\rho=$ $\left(\rho_{n, k}^{1}, \ldots, \rho_{n, k}^{k}\right)$ and $u_{n, k}=\sum_{i=1}^{k} \rho_{n, k}^{i} \phi_{i}$. Then we have

$$
\begin{gathered}
\int_{\Omega}(-\Delta)_{g_{p}}^{s} u_{n, k} \cdot \phi_{i} d x+\lambda \int_{\Omega} V(x)\left|u_{n, k}\right|^{p-2} u_{n, k} \cdot \phi_{i} d x \\
+\int_{\Omega} \frac{\left|u_{n, k}\right|^{r-1} u_{n, k}-\left|u_{n-1}\right|^{r-1} u_{n-1}}{h} \cdot \phi_{i} d x=0
\end{gathered}
$$

for all $i=1, \ldots, k$. Then, for any element $\psi \in F_{k} \subset W_{0}^{s} L_{g_{p}}(\Omega)$, we have

$$
\begin{gathered}
\int_{\Omega}(-\Delta)_{g_{p}}^{s} u_{n, k} \cdot \psi d x+\lambda \int_{\Omega} V(x)\left|u_{n, k}\right|^{p-2} u_{n, k} \cdot \psi d x \\
+\int_{\Omega} \frac{\left|u_{n, k}\right|^{r-1} u_{n, k}-\left|u_{n-1}\right|^{r-1} u_{n-1}}{h} \cdot \psi d x=0
\end{gathered}
$$


for all $i=1, \ldots, k$, i.e.,

$$
\begin{aligned}
& \int_{\Omega} \int_{\Omega} \frac{\left|u_{n, k}(x)-u_{n, k}(y)\right|^{p-2}}{|x-y|^{s(p-2)}} \frac{u_{n, k}(x)-u_{n, k}(y)}{|x-y|^{s}} \frac{\psi(x)-\psi(y)}{|x-y|^{N+s}} d x d y \\
& \quad+\lambda \int_{\Omega} V(x)\left|u_{n, k}\right|^{p-2} u_{n, k} \cdot \psi d x+\int_{\Omega} \frac{\left|u_{n, k}\right|^{r-1} u_{n, k}-\left|u_{n-1}\right|^{r-1} u_{n-1}}{h} \cdot \psi d x=0
\end{aligned}
$$

for all $i=1, \ldots, k$. Then $u_{n, k}=\sum_{i=1}^{k} \rho_{n, k}^{\tilde{i}} \phi_{i}$ is a weak solution of (3.1) on $F_{k}$ for each integer $n=1,2, \ldots, N$ and $k=1,2, \ldots$.

Lemma 3.2 (Energy estimate for finite-dimensional problem) Suppose that $\left\{u_{n, k}\right\}_{n=1,2, \ldots, N ; k=1,2, \ldots}$ is a solution of (3.1). Then, for each integer $n=1,2, \ldots, N$ and $k=$ $1,2, \ldots$, we have

$$
\begin{aligned}
\frac{r}{(r+1) h} \int_{\Omega}\left|u_{n-1}\right|^{r+1} d x \geq & \int_{\Omega} \int_{\Omega} \frac{\left|u_{n, k}(x)-u_{n, k}(y)\right|^{p}}{|x-y|^{N+s p}} d x d y \\
& +\lambda \int_{\Omega} V(x)\left|u_{n, k}\right|^{p} d x+\frac{r}{(r+1) h} \int_{\Omega}\left|u_{n, k}\right|^{r+1} d x .
\end{aligned}
$$

Proof Putting $\psi=u_{n, k}$ in (3.1), we have

$$
\begin{aligned}
& \int_{\Omega}(-\Delta)_{g_{p}}^{s} u_{n, k} \cdot u_{n, k} d x+\lambda \int_{\Omega} V(x)\left|u_{n, k}\right|^{p} d x \\
& \quad+\int_{\Omega} \frac{\left|u_{n, k}\right|^{r-1} u_{n, k}-\left|u_{n-1}\right|^{r-1} u_{n-1}}{h} \cdot u_{n, k} d x=0 .
\end{aligned}
$$

That is,

$$
\begin{aligned}
& \int_{\Omega} \frac{\left|u_{n, k}\right|^{r-1} u_{n, k}-\left|u_{n-1}\right|^{r-1} u_{n-1}}{h} \cdot u_{n, k} d x \\
& \quad=-\int_{\Omega} \int_{\Omega} \frac{\left|u_{n, k}(x)-u_{n, k}(y)\right|^{p}}{|x-y|^{N+s p}} d x d y-\lambda \int_{\Omega} V(x)\left|u_{n, k}\right|^{p} d x .
\end{aligned}
$$

By Young's inequality, we have

$$
\left|u_{n-1}\right|^{r-1} u_{n-1} \cdot u_{n, k} \leq\left|u_{n-1}\right|^{r}\left|u_{n, k}\right| \leq \frac{r}{r+1}\left|u_{n-1}\right|^{r+1}+\frac{1}{r+1}\left|u_{n, k}\right|^{r+1} .
$$

Thus we have

$$
\begin{aligned}
& \int_{\Omega} \frac{\left|u_{n, k}\right|^{r-1} u_{n, k}-\left|u_{n-1}\right|^{r-1} u_{n-1}}{h} \cdot u_{n, k} d x \\
& \quad \geq \frac{r}{(r+1) h} \int_{\Omega}\left|u_{n, k}\right|^{r+1} d x-\frac{r}{(r+1) h} \int_{\Omega}\left|u_{n-1}\right|^{r+1} d x .
\end{aligned}
$$

Combining (3.7) and (3.8), we have

$$
\begin{aligned}
\frac{r}{(r+1) h} \int_{\Omega}\left|u_{n-1}\right|^{r+1} d x \geq & \int_{\Omega} \int_{\Omega} \frac{\left|u_{n, k}(x)-u_{n, k}(y)\right|^{p}}{|x-y|^{N+s p}} d x d y \\
& +\lambda \int_{\Omega} V(x)\left|u_{n, k}\right|^{p} d x+\frac{r}{(r+1) h} \int_{\Omega}\left|u_{n, k}\right|^{r+1} d x .
\end{aligned}
$$


Thus the lemma is proved.

\section{Proof of Theorem 1.1}

In this section we prove Theorem 1.1 by showing that the limit $u_{n}=\lim _{k \rightarrow \infty} u_{n, k}$ of the subsequence $\left\{u_{n, k}\right\}_{n=1,2, \ldots, N ; k=1,2, \ldots}$ of the sequence of weak solutions of (3.1) on $F_{k}$ satisfies (1.1).

Lemma 4.1 Let $\left\{u_{n, k}\right\}_{n=1,2, \ldots, N ; k=1,2, \ldots}$ be a solution of (3.1) satisfying

$$
\begin{gathered}
\int_{\Omega}(-\Delta)_{g_{p}}^{s} u_{n, k} \cdot \psi d x+\lambda \int_{\Omega} V(x)\left|u_{n, k}\right|^{p-2} u_{n, k} \cdot \psi d x \\
+\int_{\Omega} \frac{\left|u_{n, k}\right|^{r-1} u_{n, k}-\left|u_{n-1}\right|^{r-1} u_{n-1}}{h} \cdot \psi d x=0
\end{gathered}
$$

$\forall \psi \in F_{k}$. Then there exists a subsequence, up to a subsequence, $\left\{u_{n, k}\right\}_{n=1,2, \ldots, N ; k=1,2, \ldots}$ converging to $\lim _{k \rightarrow \infty} u_{n, k}=u_{n}$ such that

(i) $u_{n, k} \rightarrow u_{n}$ strongly as $k \rightarrow \infty$ in $L_{g_{r}}(\Omega)$.

(ii) $\left|u_{n, k}\right|^{r-1} u_{n, k} \rightarrow\left|u_{n}\right|^{r-1} u_{n}$ strongly as $k \rightarrow \infty$ in $L^{1}(\Omega)$.

Proof (i) The sequence $u_{n-1} \in L_{g_{r+1}}(\Omega)$ is defined inductively and by Lemma 3.2, $\left\{u_{n, k}\right\}$ is bounded in $W_{0}^{s} L_{g_{p}}(\Omega)$. Since the embedding $W_{0}^{s} L_{g_{p}}(\Omega) \hookrightarrow L_{g_{q}}(\Omega)$ is continuous and compact for any $q$ with $1 \leq q<\frac{N p}{N-p}=r+1$, the embedding $W_{0}^{s} L_{g_{p}}(\Omega) \hookrightarrow L_{g_{r}}(\Omega)$ is continuous and compact. Thus the sequence $\left\{u_{n, k}\right\}$ has a subsequence, up to a subsequence, $\left\{u_{n, k}\right\}$ converging strongly to $\lim _{k \rightarrow \infty 0} u_{n, k}=u_{n}$ in $L_{g_{r}}(\Omega)$.

(ii) By Lemma 2.7 (i), there exist constants $C>0$ and $C^{\prime}>0$ such that

$$
\begin{aligned}
\left.\int_{\Omega}|| u_{n, k}\right|^{r-1} u_{n, k}-\left|u_{n}\right|^{r-1} u_{n} \mid d x & \leq C \int_{\Omega}\left(\left|u_{n, k}\right|^{r-1}+\left|u_{n}\right|^{r-1}\right)\left|u_{n, k}-u_{n}\right| d x \\
& \leq C\left(\int_{\Omega}\left(\left|u_{n, k}\right|^{r}+\left|u_{n}\right|^{r}\right) d x\right)^{\frac{r-1}{r}}\left(\int_{\Omega}\left|u_{n, k}-u_{n}\right|^{r} d x\right)^{\frac{1}{r}} \\
& \leq C^{\prime}\left\|u_{n, k}-u_{n}\right\|_{L_{g r}} .
\end{aligned}
$$

Since by (i) $u_{n, k} \rightarrow u_{n}$ strongly as $k \rightarrow \infty$ in $L_{g_{r}}(\Omega)$ and $u_{n} \in L_{g_{r}}(\Omega)$, it follows that $\left|u_{n, k}\right|^{r-1} u_{n, k}-\left|u_{n}\right|^{r-1} u_{n} \in L^{1}(\Omega)$.

Proof of Theorem 1.1 By Lemma 3.1, for each $n=1,2, \ldots, N$ and $k=1,2, \ldots$, there exists a unique weak solution $u_{n, k} \in F_{k} \subset W_{0}^{s} L_{g_{p}}(\Omega)$ of (3.1). By Lemma 4.1, there exists a subsequence, up to a subsequence, $\left\{u_{n, k}\right\}$ converging strongly to $\lim _{k \rightarrow \infty} u_{n, k}=u_{n}$ in $L_{g_{r}}(\Omega)$.

We shall show that $u_{n}$ satisfies (1.1). That is, we shall show that, for any $w \in W_{0}^{s} L_{g_{p}}(\Omega)$,

$$
\begin{gathered}
\int_{\Omega}(-\Delta)_{g_{p}}^{s} u_{n} \cdot w d x+\lambda \int_{\Omega} V(x)\left|u_{n}\right|^{p-2} u_{n} \cdot w d x \\
+\int_{\Omega} \frac{\left|u_{n}\right|^{r-1} u_{n}-\left|u_{n-1}\right|^{r-1} u_{n-1}}{h} \cdot w d x=0
\end{gathered}
$$


i.e.,

$$
\begin{aligned}
& \int_{\Omega} \int_{\Omega} \frac{\left|u_{n}(x)-u_{n}(y)\right|^{p-2}}{|x-y|^{s(p-2)}} \frac{u_{n}(x)-u_{n}(y)}{|x-y|^{s}} \frac{w(x)-w(y)}{|x-y|^{N+s}} d x d y \\
& \quad+\lambda \int_{\Omega} V(x)\left|u_{n}\right|^{p-2} u_{n} \cdot w d x+\int_{\Omega} \frac{\left|u_{n}\right|^{r-1} u_{n}-\left|u_{n-1}\right|^{r-1} u_{n-1}}{h} \cdot w d x=0 .
\end{aligned}
$$

In fact, for any $w \in W_{0}^{s} L_{g_{p}}(\Omega)$, let $w_{k}=\sum_{i=1}^{k} h_{n, i} \phi_{i}(x)$ be the approximating sequence which converges to $w$ in $W_{0}^{s} L_{g_{p}}(\Omega)$. By Lemma 2.7 (ii), there exists a constant $C_{2}>0$ such that

$$
\begin{aligned}
\int_{\Omega} & \left((-\Delta)_{g_{p}}^{s} w_{k}-(-\Delta)_{p}^{s} u_{n, k}\right) \cdot\left(w_{k}-u_{n, k}\right) d x \\
= & \int_{\Omega} \int_{\Omega} \frac{\left(\left|w_{k}(x)-w_{k}(y)\right|^{p-2}\left(w_{k}(x)-w_{k}(y)\right)-\left(\left|u_{n, k}(x)-u_{n, k}(y)\right|^{p-2}\left(u_{n, k}(x)-u_{n, k}(y)\right)\right.\right.}{|x-y|^{s(p-2)}|x-y|^{s}} \\
& \quad \cdot \frac{\left(w_{k}(x)-u_{n, k}(x)\right)-\left(w_{k}(y)-u_{n, k}(y)\right)}{|x-y|^{N+s}} d x d y \\
\geq & C_{2} \int_{\Omega} \int_{\Omega} \frac{\left|\left(w_{k}(x)-u_{n, k}(x)\right)-\left(w_{k}(y)-u_{n, k}(y)\right)\right|^{p}}{|x-y|^{N+s p}} d x d y \geq 0 .
\end{aligned}
$$

On the other hand, putting $w=u_{n, k}$ in (3.1), we have

$$
\begin{aligned}
-\int_{\Omega}(-\Delta)_{g_{p}}^{s} u_{n, k} \cdot u_{n, k} d x= & \lambda \int_{\Omega} V(x)\left|u_{n, k}\right|^{p-2} u_{n, k} \cdot u_{n, k} d x \\
& +\int_{\Omega} \frac{\left|u_{n, k}\right|^{r-1} u_{n, k}-\left|u_{n-1}\right|^{r-1} u_{n-1}}{h} \cdot u_{n, k} d x .
\end{aligned}
$$

Taking the test function as $w_{k}-u_{n, k}$ in (3.1), we have

$$
\begin{aligned}
& -\int_{\Omega}(-\Delta)_{g_{p}}^{s} u_{n, k} \cdot\left(w_{k}-u_{n, k}\right) d x \\
& =\lambda \int_{\Omega} V(x)\left|u_{n, k}\right|^{p-2} u_{n, k} \cdot\left(w_{k}-u_{n, k}\right) d x \\
& \quad+\int_{\Omega} \frac{\left|u_{n, k}\right|^{r-1} u_{n, k}-\left|u_{n-1}\right|^{r-1} u_{n-1}}{h} \cdot\left(w_{k}-u_{n, k}\right) d x .
\end{aligned}
$$

By adding (4.1) and (4.2), we have

$$
\begin{aligned}
& \int_{\Omega}\left((-\Delta)_{g_{p}}^{s} w_{k} \cdot\left(w_{k}-u_{n, k}\right)+\lambda \int_{\Omega} V(x)\left|u_{n, k}\right|^{p-2} u_{n, k} \cdot\left(w_{k}-u_{n, k}\right) d x\right. \\
& \quad+\int_{\Omega} \frac{\left|u_{n, k}\right|^{r-1} u_{n, k}-\left|u_{n-1}\right|^{r-1} u_{n-1}}{h} \cdot\left(w_{k}-u_{n, k}\right) d x \geq 0 .
\end{aligned}
$$

By the energy estimate theorem Lemma 3.2, there exists a constant $C_{1}>0$ such that

$$
\begin{aligned}
& \int_{\Omega}(-\Delta)_{g_{p}}^{s} u_{n, k} \cdot u_{n, k} d x+\lambda \int_{\Omega} V(x)\left|u_{n, k}\right|^{p} d x+\frac{r}{(r+1) h} \int_{\Omega}\left|u_{n, k}\right|^{r+1} d x \\
& \quad=\int_{\Omega} \int_{\Omega} \frac{\left|u_{n, k}(x)-u_{n, k}(y)\right|^{p}}{|x-y|^{N+s p}} d x d y+\lambda \int_{\Omega} V(x)\left|u_{n, k}\right|^{p} d x+\frac{r}{(r+1) h} \int_{\Omega}\left|u_{n, k}\right|^{r+1} d x \\
& \quad \leq C_{1},
\end{aligned}
$$


it follows that the sequence $\left\{u_{n, k}\right\}$ is bounded in $L_{g_{p}}(\Omega)$ and so, up to a subsequence,

$u_{n, k}$ converges to $u_{n}$ weakly in $L_{g_{p}}(\Omega)$.

Passing to the limit as $k \rightarrow \infty$, we have that the first part and the second part of the lefthand side of (4.4)

$$
\begin{gathered}
\int_{\Omega}\left((-\Delta)_{g_{p}}^{s} w_{k} \cdot\left(w_{k}-u_{n, k}\right)+\lambda \int_{\Omega} V(x)\left|u_{n, k}\right|^{p-2} u_{n, k} \cdot\left(w_{k}-u_{n, k}\right) d x\right. \\
\longrightarrow \int_{\Omega}\left((-\Delta)_{g_{p}}^{s} w \cdot\left(w-u_{n}\right)+\lambda \int_{\Omega} V(x)\left|u_{n}\right|^{p-2} u_{n} \cdot\left(w-u_{n}\right) d x\right.
\end{gathered}
$$

On the other hand, by (ii) of Lemma $4.1, u_{n, k} \rightarrow u_{n}$ a.e., in $\Omega$ and by Vitali's converging theorem, up to a subsequence,

$$
\left|u_{n, k}\right|^{r-1} u_{n, k} \text { converges to }\left|u_{n}\right|^{r-1} u_{n} \text { weakly in } L^{\frac{r+1}{r}}(\Omega) \text {. }
$$

It follows that the third part of the left-hand side of (4.4)

$$
\begin{gathered}
\int_{\Omega} \frac{\left|u_{n, k}\right|^{r-1} u_{n, k}-\left|u_{n-1}\right|^{r-1} u_{n-1}}{h} \cdot\left(w_{k}-u_{n, k}\right) d x \\
\quad \rightarrow \int_{\Omega} \frac{\left|u_{n}\right|^{r-1} u_{n}-\left|u_{n-1}\right|^{r-1} u_{n-1}}{h} \cdot\left(w-u_{n}\right) d x
\end{gathered}
$$

Combining (4.4), (4.5), and (4.6), we have

$$
\begin{gathered}
\int_{\Omega}\left((-\Delta)_{g_{p}}^{s} w \cdot\left(w-u_{n}\right)+\lambda \int_{\Omega} V(x)\left|u_{n}\right|^{p-2} u_{n} \cdot\left(w-u_{n}\right) d x\right. \\
+\int_{\Omega} \frac{\left|u_{n}\right|^{r-1} u_{n}-\left|u_{n-1}\right|^{r-1} u_{n-1}}{h} \cdot\left(w-u_{n}\right) d x \geq 0
\end{gathered}
$$

for any $w \in W_{0}^{s} L_{g_{p}}(\Omega)$. Let us set

$$
w=u_{n}+\eta \psi \quad \text { for any } \psi \in W_{0}^{s} L_{g_{p}}(\Omega) \text { and } \eta>0 \text {. }
$$

Inserting the test function $w=u_{n}+\eta \psi$ in (4.7), we have

$$
\begin{gathered}
\int_{\Omega}\left((-\Delta)_{g_{p}}^{s}\left(u_{n}+\eta \psi\right) \cdot \psi+\lambda \int_{\Omega} V(x)\left|u_{n}\right|^{p-2} u_{n} \cdot \psi d x\right. \\
+\int_{\Omega} \frac{\left|u_{n}\right|^{r-1} u_{n}-\left|u_{n-1}\right|^{r-1} u_{n-1}}{h} \cdot \psi d x \geq 0
\end{gathered}
$$

Letting $\eta \rightarrow 0$, we have

$$
\begin{gathered}
\int_{\Omega}\left((-\Delta)_{g_{p}}^{s}\left(u_{n}\right) \cdot \psi+\lambda \int_{\Omega} V(x)\left|u_{n}\right|^{p-2} u_{n} \cdot \psi d x\right. \\
+\int_{\Omega} \frac{\left|u_{n}\right|^{r-1} u_{n}-\left|u_{n-1}\right|^{r-1} u_{n-1}}{h} \cdot \psi d x \geq 0 .
\end{gathered}
$$


Replacing $\psi$ by $-\psi$ and letting $\eta \rightarrow 0$ in (4.8), we have

$$
\begin{gathered}
\int_{\Omega}\left((-\Delta)_{g_{p}}^{s}\left(u_{n}\right) \cdot \psi+\lambda \int_{\Omega} V(x)\left|u_{n}\right|^{p-2} u_{n} \cdot \psi d x\right. \\
+\int_{\Omega} \frac{\left|u_{n}\right|^{r-1} u_{n}-\left|u_{n-1}\right|^{r-1} u_{n-1}}{h} \cdot \psi d x \leq 0 .
\end{gathered}
$$

Combining (4.9) and (4.10), we obtain the equality

$$
\begin{gathered}
\int_{\Omega}\left((-\Delta)_{g_{p}}^{s}\left(u_{n}\right) \cdot \psi+\lambda \int_{\Omega} V(x)\left|u_{n}\right|^{p-2} u_{n} \cdot \psi d x\right. \\
+\int_{\Omega} \frac{\left|u_{n}\right|^{r-1} u_{n}-\left|u_{n-1}\right|^{r-1} u_{n-1}}{h} \cdot \psi d x=0 .
\end{gathered}
$$

Thus the proof of Theorem 1.1 is complete.

\section{Acknowledgements}

Not applicable.

\section{Funding}

Q-Heung Choi was supported by the Basic Science Research Program through the National Research Foundation of Korea(NRF) funded by the Ministry of Education, Science and Technology (NRF-2017R1D1A1B03030024). Tacksun Jung was supported by the Basic Science Research Program through the National Research Foundation of Korea(NRF) funded by the Ministry of Science, ICT and Future Planning (NRF-2017R1A2B4005883).

\section{Abbreviations}

Not applicable.

\section{Availability of data and materials}

Not applicable.

\section{Competing interests}

The authors declare that they have no competing interests.

\section{Authors' contributions}

QHCh introduced the main ideas of multiplicity study for this problem. TJ participated in applying the method for solving this problem and drafted the manuscript. All authors contributed equally to reading and approving the final manuscript.

\section{Author details}

'Department of Mathematics Education, Inha University, Incheon 402-751, Korea. ${ }^{2}$ Department of Mathematics, Kunsan National University, Kunsan 573-701, Korea.

\section{Publisher's Note}

Springer Nature remains neutral with regard to jurisdictional claims in published maps and institutional affiliations.

Received: 13 September 2020 Accepted: 10 February 2021 Published online: 26 February 2021

\section{References}

1. Adams, D.R., Hedberg, L.I.: Function Spaces and Potential Theory. Grundlehren der Mathematischen Wissenschaften (Fundamental Principles of Mathematical Sciences), vol. 314. Springer, Berlin (1996)

2. Alves, C.O., Ferreira, M.C.: Existence of solutions for a class of $p(x)$-Laplacian equations involving a concave-convex nonlinearity with critical growth in $R^{N}$. Topol. Methods Nonlinear Anal. 45, 399-422 (2015)

3. Autuori, G. Pucci, P. Elliptic problems involving the fractional Laplacian in $R^{N}$.J. Differ. Equ. 255, 2340-2362 (2013)

4. Azroul, E., Benkirane, A., Srati, M.: Existence of solutions for a nonlocal type problem in fractional Orlicz Sobolev spaces. Preprint (2019)

5. Bahrouni, A.: Comparison and sub-supsolution principles for the fractional $p(x)$-Laplacian. J. Math. Anal. Appl. 458, 1363-1372 (2018)

6. Bahrouni, A., Radulescu, V:: On a new fractional Sobolev space and application to nonlocal variational problems with variable exponent. Discrete Contin. Dyn. Syst., Ser. S 11, 379-389 (2018)

7. Bonder, J.F., Salort, A.M.: Fractional order Orlicz-Sobolev spaces. J. Funct. Anal. (2019). https://doi.org/10.1016/j.jfa.2019.04.003

8. Caffarelli, L., Silvestre, L.: An extension problem related to the fractional Laplacian. Commun. Partial Differ. Equ. 32 $1245-1260(2007)$ 
9. Chen, Y., Levine, S., Rao, M.: Variable exponent, linear growth functionals in image restoration. SIAM J. Appl. Math. 66, 1383-1406 (2006)

10. Di Nezza, E., Palatucci, G., Valdinoci, E.: Hitchhiker's guide to the fractional Sobolev spaces. Bull. Sci. Math. 136, 521-573 (2012)

11. Diening, L., Harjulehto, P., Hästö, P., Ružička, M.: Lebesgue and Sobolev Spaces with Variable Exponents. Springe, Heidelberg (2011)

12. Fărcăseanu, M.: On an eigenvalue problem involving the fractional (s, p)-Laplacian. Fract. Calc. Appl. Anal. 21, 94-103 (2018)

13. Fărcăseanu, M., Mihăilescu, M., Stancu-Dumitru, D.: Perturbed fractional eigenvalue problems. Discrete Contin. Dyn. Syst., Ser. A 37, 6243-6255 (2017)

14. Fiscella, A.: A fractional Kirchhoff problem involving a singular term and a critical nonlinearity. Adv. Nonlinear Anal. 8, 645-660 (2019)

15. Ji, C., Fang, F., Zhang, B.: A multiplicity result for asymptotically linear Kirchhoff equations. Adv. Nonlinear Anal. 8 , 267-277 (2019)

16. Kaufmann, U., Rossi, J., Vidal, R.: Fractional Sobolev spaces with variable exponents and fractional $p(x)$-Laplacians. Electron. J. Qual. Theory Differ. Equ. 76, 1 (2017)

17. Kikuchi, K., Negoro, A.: On Markov processes generated by pseudodifferential operator of variable order. Osaka J. Math. 34, 319-335 (1997)

18. Leopold, H.G.: Embedding of function spaces of variable order of differentiation. Czechoslov. Math. J. 49, 633-641 (1999)

19. Lindgren, E., Lindqvist, P.: Fractional eigenvalues. Calc. Var. Partial Differ. Equ. 49, 795-826 (2014)

20. Lorenzo, C.F., Hartley, T.T.: Initialized fractional calculus. Int. J. Appl. Math. 3, 249-265 (2000)

21. Lorenzo, C.F., Hartley, T.T.: Variable order and distributed order fractional operators. Nonlinear Dyn. 29, 57-98 (2002)

22. Mihăilescu, M., Pucci, P., Rădulescu, V.: Eigenvalue problems for anisotropic quasilinear elliptic equations with variable exponent. J. Math. Anal. Appl. 340, 687-698 (2008)

23. Mihăilescu, M., Rădulescu, V.: A multiplicity result for a nonlinear degenerate problem arising in the theory of electrorheological fluids. Proc. R. Soc. Lond., Ser. A, Math. Phys. Eng. Sci. 462, 2625-2641 (2006)

24. Mingqi, X., Molica Bisci, G., Tian, G., Zhang, B.: Infinitely many solutions for the stationary Kirchhoff problems involving the fractional $p$-Laplacian. Nonlinearity 29, 357-374 (2016)

25. Mingqi, X., Rădulescu, V.D., Zhang, B.: Fractional Kirchhoff problems with critical Trudinger-Moser nonlinearity. Calc Var. Partial Differ. Equ. 58, 57 (2019)

26. Mingqi, X., Rădulescu, V.D., Zhang, B.: Nonlocal Kirchhoff problems with singular exponential nonlinearity. Appl. Math Optim. (2020). https://doi.org/10.1007/s00245-020-09666-3

27. Molica Bisci, G., Rădulescu, V., Servadei, R.: Variational Methods for Nonlocal Fractional Problems. Encyclopedia of Mathematics and Its Applications, vol. 162. Cambridge University Press, Cambridge (2016)

28. Pucci, P., Xiang, M., Zhang, B.: Multiple solutions for nonhomogeneous Schrödinger-Kirchhoff type equations involving the fractional $p$-Laplacian in $R^{N}$. Calc. Var. Partial Differ. Equ. 54, 2785-2806 (2015)

29. Pucci, P., Xiang, M., Zhang, B.: Existence and multiplicity of entire solutions for fractional $p$-Kirchhoff equations. Adv. Nonlinear Anal. 5, 27-55 (2016)

30. Ruiz-Medina, M.D., Anh, V.V., Angulo, J.M.: Fractional generalized random fields of variable order. Stoch. Anal. Appl. 22 775-799 (2004)

31. Ružička, M.: Electrorheological Fluids: Modeling and Mathematical Theory. Springer, Berlin (2002)

32. Salort, A.M.: A fractional Orlicz-Sobolev eigenvalue problem and related Hardy inequalities. arXiv:e-prints, arXiv:1807.03209 (2018)

33. Servadei, R., Valdinoci, E.: Mountain pass solutions for non-local elliptic operator. J. Math. Anal. Appl. 389, 887-898 (2012)

34. Servadei, R., Valdinoci, E.: Variational methods for non-local operators of elliptic type. Discrete Contin. Dyn. Syst. 33, 2105-2137 (2013)

35. Xiang, M., Rădulescu, V., Zhang, B.: Existence of solutions for perturbed fractional $p$-Laplacian equations. J. Differ. Equ. 260, 1392-1413 (2016)

36. Xiang, M., Wang, F.: Fractional Schrödinger-Poisson-Kirchhoff systems involving critical nonlinearities. Nonlinear Anal. $164,1-26(2017)$

37. Xiang, M., Zhang, B., Ferrara, M.: Existence of solutions for Kirchhoff type problem involving the non-local fractional p-Laplacian. J. Math. Anal. Appl. 424, 1021-1041 (2015)

38. Xiang, M., Zhang, B., Guo, X.: Infinitely many solutions for a fractional Kirchhoff type problem via Fountain theorem. Nonlinear Anal. 120, 299-313 (2015)

39. Xiang, M., Zhang, B., Rădulescu, V.D.: Superlinear Schrödinger-Kirchhoff type problems involving the fractional p-Laplacian and critical exponent. Adv. Nonlinear Anal. 9, 690-709 (2020)

40. Zhikov, V.V.: Averaging of functionals of the calculus of variations and elasticity theory. Izv. Akad. Nauk SSSR, Ser. Mat. 50(4), 675-710 (1986). English transl., Math. USSR, Izv. 29(1), 33-66 (1987) 